\title{
Fruit and vegetable consumption is inversely associated with blood pressure in a Mediterranean population with a high vegetable-fat intake: the Seguimiento Universidad de Navarra (SUN) Study
}

\author{
Alvaro Alonso ${ }^{1,2}$, Carmen de la Fuente ${ }^{1}$, Ana M. Martín-Arnau ${ }^{1}$, Jokin de Irala ${ }^{1}$, J. Alfredo Martínez ${ }^{3}$ \\ and Miguel Ángel Martínez-González ${ }^{1 *}$ \\ ${ }^{1}$ Department of Epidemiology and Public Health, University of Navarra, Irunlarrea 1, E-31008 Pamplona, Spain \\ ${ }^{2}$ Preventive Medicine and Quality Management Service, Virgen del Camino Hospital, Pamplona, Spain \\ ${ }^{3}$ Department of Physiology and Nutrition, University of Navarra, Pamplona, Spain
}

(Received 5 December 2003 - Revised 16 March 2004 - Accepted 12 April 2004)

\begin{abstract}
There is evidence that a diet rich in fruit and vegetables reduces blood pressure (BP). Characteristically, the Mediterranean diet is rich in plant-derived foods and also in fat, but studies conducted in Mediterranean countries to relate diet to BP are scarce. We studied the association between fruit and vegetable consumption and BP in a cross-sectional analysis of 4393 participants in the Seguimiento Universidad de Navarra (SUN) Study, an ongoing dynamic cohort study in Spain. Diet was measured using a food-frequency questionnaire previously validated in Spain. Fat represented more than $37 \%$ total energy intake. Subjects were considered to have undiagnosed hypertension if they reported systolic $\mathrm{BP} \geq 140 \mathrm{mmHg}$ or diastolic $\mathrm{BP} \geq 90 \mathrm{mmHg}$, and not a medical diagnosis of hypertension. The adjusted prevalence odds ratio of undiagnosed hypertension (upper $v$. lowest quintile) was 0.58 (95\% CI $0.36,0.91 ; P$ for trend $0 \cdot 01$ ) for vegetable consumption and 0.68 (95\% CI 0.43, 1.09; $P$ for trend 0.10$)$ for fruit consumption. Comparing those in the highest quintile of both fruit and vegetable consumption with those in the lowest quintile of both food groups, the prevalence odds ratio was 0.23 (95\% CI $0 \cdot 10$, 0.55 ; $P=0.001$ ), after adjusting for risk factors for hypertension and other dietary exposures. In a Mediterranean population with an elevated fat consumption, a high fruit and vegetable intake is inversely associated with BP levels.
\end{abstract}

Hypertension: Plant foods: Mediterranean diet

There is increasing evidence that certain lifestyles can lead to compelling reductions in blood pressure (BP) levels and in the prevalence of hypertension, resulting in a decreased risk of its complications. Maintaining a normal body weight, engaging in customary physical activity, and reducing $\mathrm{Na}$ and alcohol intake, are the most classical and typical lifestyle recommendations for the primary prevention of hypertension (Whelton et al. 2002).

There are also other aspects concerning dietary patterns presumably related to BP levels, such as $\mathrm{K}, \mathrm{Ca}, \mathrm{Mg}$, fibre, protein and fat intake (Hermansen, 2000). Nonetheless, the most appealing reductions in BP have been related to the modification of whole diets and not with the administration of separated nutrient supplements, as shown by the Dietary Approaches to Stop Hypertension (DASH) trial (Appel et al. 1997; Sacks et al. 2001). In the DASH trial, a diet rich in fruit, vegetables and low-fat dairy products, but reduced in saturated and total fat, obtained an average $5.5 \mathrm{mmHg}$ reduction in systolic BP (SBP).

The classical Mediterranean diet has been postulated as a protective pattern against CHD (Willett et al. 1995; De Lorgeril et al. 1999; Robertson \& Smaha, 2001; Singh et al. 2002; Martínez-González et al. 2003; Trichopoulou et al. 2003; Martínez-González \& Sánchez-Villegas, 2004). In addition to a high vegetable-fat content, mainly from MUFA (with olive oil as the main source), another major component of this dietary pattern is an abundant consumption of vegetables and fruits. This could be one of the multiple pathways by which the cardioprotective effect of a Mediterranean dietary pattern might be elicited.

The aim of the present study was to evaluate the relationship between fruit and vegetable consumption and BP levels at baseline in the Seguimiento Universidad de Navarra (SUN) Study, a dynamic cohort of university graduates intended to evaluate the effect of a Mediterranean dietary pattern on the risk of cardiovascular disease.

\section{Methods \\ The Seguimiento Universidad de Navarra Study}

The SUN project was designed in collaboration with the Harvard School of Public Health and its methodology is similar to that used in large American cohorts such as

\footnotetext{
Abbreviations: BP, blood pressure; DASH, Dietary Approaches to Stop Hypertension; DBP, diastolic blood pressure; MET, metabolic equivalent; OR, odds ratio; SBP, systolic blood pressure; SFA, saturated fatty acid; SUN, Seguimiento Universidad de Navarra.

* Corresponding author: Dr Miguel Angel Martínez-González, fax +34948 4256 49, email mamartinez@unav.es
} 
the Nurses' Health Study and the Health Professionals Follow-up Study. A detailed description of its methods has been published elsewhere (Martínez-González et al. 2002; Sánchez-Villegas et al. 2003).

\section{Study population}

The recruitment of the cohort started in December 1999 and it is still ongoing because the design is that of a dynamic cohort study (Rothman, 2002). A mailed questionnaire was sent to all alumni of the University of Navarra living in Spain, to highly educated subjects belonging to an Insurance Company (ACUNSA) and to registered nurses from the Professional Association of Nurses of Navarra. The study protocol has been approved by the Institutional Review Board of the University of Navarra. We considered the response to the initial questionnaire as an informed consent to participate in the study.

For the present analysis, we have taken into account the first 8830 participants of the cohort. Subjects reporting a diagnosis of cancer, CHD or diabetes were excluded because of possible dietary changes following the diagnosis of these disorders, thus originating a reverse causation bias, typical in cross-sectional studies (Tormo et al. 2000). We also excluded those participants reporting a medical diagnosis of hypertension including those taking anti-hypertensive drugs. Those who did not report their usual BP were also excluded. These exclusion criteria left 4393 participants available for analysis.

\section{Dietary assessment}

Diet was assessed using a semi-quantitative food-frequency questionnaire previously validated in Spain (MartínMoreno et al. 1993). The current version of this questionnaire covers 136 individual food items. For each food, a standard portion size was specified and participants were asked to estimate their average intake over the previous year, choosing between nine categories from 'never or almost never' to 'more than six times a day'. There were thirteen separate items for different fruits, and eleven items for vegetable consumption.

\section{Other covariates}

The baseline survey included questions about different socio-demographic factors (sex, age, marital and employment status, university degree), anthropometric (weight, height), health-related habits (smoking status, alcohol consumption, physical activity) and clinical variables (use of medication, personal and family history of CHD, cancer and other diseases). BMI was calculated as the selfreported weight in kilograms divided by the square of height in metres.

To quantify the amount of activity during leisure time, an activity metabolic equivalent (MET) index was computed by assigning a multiple of resting metabolic rate (MET score) to each activity. Time spent in each of the activities was multiplied by the MET score specific to each activity, and then summed over all activities obtaining a value of overall weekly MET-hours (Martínez-González et al. 1999; Ainsworth et al. 2000).

\section{Assessment of blood pressure}

BP status was defined by self-reported responses to the questionnaire. Participants were asked whether they had ever had a medical diagnosis of hypertension. Those who had a medical diagnosis or were under treatment for hypertension were excluded from the analyses. In addition, we requested information from all participants about their usual SBP and diastolic BP (DBP) levels. The participants could choose between the following categories for SBP (mmHg): lower than 100; 101-110; 111-120; 121-130; $131-140 ; 141-150 ; 151-160 ; 161-175$; greater than 175. For DBP they could choose between $(\mathrm{mmHg})$ : lower than 60; 61-70; 71-80; 81-90; 91-100; $101-110 ; 111-120 ; 121-130 ;$ greater than 130 . No specific recommendations about timing or device for BP measurements were given. We considered an individual to have previously undiagnosed hypertension if she or he did not report a medical diagnosis or treatment for hypertension, but nevertheless reported that she or he had SBP equal to or greater than $140 \mathrm{mmHg}$ and/or DBP equal to or greater than $90 \mathrm{mmHg}$. We followed the criteria from the seventh report of the Joint National Committee (Chobanian et al. 2003). This case-definition allowed us to capture mainly recent cases of hypertension, excluding those who might have been labelled as 'hypertensive' for a period before our assessment.

\section{Statistical analysis}

The main outcome for analysis was previously undiagnosed hypertension according to the criteria defined earlier. Nutrients were derived using Spanish food-composition tables (Mataix Verdú, 2003). Food consumption was adjusted for total energy intake using the residuals method (Willett, 1998). For each energy-adjusted food, we specified five levels of intake with quintiles as cut-off points. We calculated prevalence ratios of undiagnosed hypertension associated with food intakes, defined as the prevalence of undiagnosed hypertension among subjects in each category of food intake divided by the corresponding rate among subjects in the lowest category of intake. To assess the combined effect of fruit and vegetable intake, we created a new variable summing up quintiles of fruit and vegetables. In this manner, we obtained a variable with a range from 2 to 10 (scoring 2 for those in the lowest quintile of both fruit and vegetable consumption, and 10 for those in the upper quintile of both food groups). To adjust for several variables simultaneously, we used non-conditional logistic regression, obtaining in this way prevalence odds ratios (OR). In an additional analysis, we used self-reported SBP and DBP as continuous variables, and multiple linear regression analyses were performed. All these analyses were repeated considering selfreported hypertension or taking anti-hypertensive medication as the dependent variable. Finally, we conducted logistic regression analyses after stratifying by MUFA intake to assess possible effect modification by this 
variable. We tested the significance of the interaction term with a likelihood ratio test (Hosmer \& Lemeshow, 2000). All analyses were performed with SPSS 10.0 for Windows (SPSS Inc., Chicago, IL, USA).

\section{Results}

To prevent biases derived from reverse causation (prescribed diets as a consequence of a diagnosis of cardiovascular disease, diabetes or hypertension) and also to preclude any misclassification due to the imputation of missing values, we excluded those participants reporting a diagnosis of cancer or cardiovascular disease ( $n$ 619), diabetes ( $n$ 108) or a medical diagnosis of hypertension ( $n$ 546). Additionally, 546 subjects were excluded from the analyses due to incomplete information about diet. BP levels were unknown for a sizeable number of participants, because 2555 subjects did not report their BP level. Finally, sixty-three participants lacked information in other covariates, thus resulting in 4393 individuals available for analysis. Table 1 reports the distribution of selected variables among individuals excluded and individuals included in the analyses. A lower mean fruit and vegetable consumption was found among participants with missing values for BP. In addition, they were younger, had a higher intake of alcohol, of total fat and saturated fats, and a lower intake of MUFA. Therefore, individuals who lacked information about this variable perhaps were more likely to include more cases of hypertension.

The mean SBP and DBP were 114 and $70 \mathrm{mmHg}$, respectively. According to their reported SBP and DBP, $217(4.9 \%)$ participants were considered to have previously undiagnosed hypertension. The distribution of known risk factors according to quintiles of vegetable and fruit consumption is shown in Tables 2 and 3. Age and female sex were positively correlated with fruit and vegetable consumption, whereas alcohol and $\mathrm{Na}$ intakes were negatively correlated with both food groups. The BMI and the leisure-time physical activity were not related to the consumption of these foods. Total fat intake was inversely correlated with fruit and vegetable consumption. This is mainly due to saturated fatty acid (SFA) intake, whose intake decreased with a higher fruit and vegetable consumption. On the other hand, MUFA intake did not change with vegetable consumption.

In the age- and sex-adjusted analyses, consumption of vegetables and fruits showed an inverse linear relationship with the prevalence of undiagnosed hypertension (Tables 4 and 5). After adjustment for known risk factors for hypertension, the relationship between vegetables and

Table 1. Distribution of selected variables among participants with self-reported medical conditions, participants with missing values for blood pressure, and participants included in the analyses

(Mean values)

\begin{tabular}{|c|c|c|c|}
\hline & $\begin{array}{l}\text { Subjects with self-reported } \\
\text { medical conditions }\end{array}$ & $\begin{array}{l}\text { Subjects with missing values } \\
\text { for blood pressure }\end{array}$ & $\begin{array}{l}\text { Subjects included } \\
\text { in the analysis }\end{array}$ \\
\hline Participants $(n)$ & 1273 & 2555 & 4393 \\
\hline Median fruit consumption (g/d) & 235.4 & $107 \cdot 8$ & $262 \cdot 6$ \\
\hline Age (years) & $51 \cdot 3$ & $36 \cdot 4$ & $38 \cdot 3$ \\
\hline Sex (\% female) & $43 \cdot 7$ & $55 \cdot 0$ & $65 \cdot 8$ \\
\hline BMI $\left(\mathrm{kg} / \mathrm{m}^{2}\right)$ & $25 \cdot 4$ & $23 \cdot 1$ & $23 \cdot 1$ \\
\hline Energy-adjusted alcohol consumption (g/d) & $13 \cdot 1$ & $7 \cdot 1$ & $6 \cdot 3$ \\
\hline Energy-adjusted Na consumption (mg/d) & 3783 & 4676 & 4258 \\
\hline Total fat intake (\% of energy intake) & $36 \cdot 0$ & $37 \cdot 3$ & $37 \cdot 1$ \\
\hline MUFA intake ( $\%$ of energy intake) & $15 \cdot 6$ & $15 \cdot 7$ & $16 \cdot 0$ \\
\hline Saturated fatty acids intake (\% of energy intake) & $12 \cdot 1$ & $13 \cdot 3$ & $12 \cdot 6$ \\
\hline
\end{tabular}

Table 2. Distribution of selected variables across quintiles (Q) of energy-adjusted vegetable consumption in the Seguimiento Universidad de Navarra (SUN) Study

(Mean values)

\begin{tabular}{|c|c|c|c|c|c|}
\hline & Q1 (lowest) & Q2 & Q3 & Q4 & Q5 (highest) \\
\hline Participants $(n)$ & 878 & 879 & 880 & 878 & 878 \\
\hline Median vegetable consumption (g/d) & 240 & 343 & 452 & 608 & 957 \\
\hline Sex (\% female) & 49 & 62 & 65 & 72 & 79 \\
\hline BMI $\left(\mathrm{kg} / \mathrm{m}^{2}\right)$ & $23 \cdot 3$ & $23 \cdot 2$ & $23 \cdot 3$ & $23 \cdot 0$ & $22 \cdot 8$ \\
\hline Physical activity during leisure time in metabolic equivalents (h/week) & $19 \cdot 9$ & $19 \cdot 0$ & $19 \cdot 5$ & $20 \cdot 4$ & $20 \cdot 7$ \\
\hline Energy-adjusted alcohol consumption (g/d) & $7 \cdot 3$ & $6 \cdot 0$ & $6 \cdot 9$ & $5 \cdot 8$ & $5 \cdot 3$ \\
\hline Total fat intake (\% of energy intake) & $37 \cdot 7$ & $38 \cdot 0$ & $36 \cdot 9$ & $37 \cdot 1$ & $35 \cdot 5$ \\
\hline MUFA intake ( $\%$ of energy intake) & $15 \cdot 9$ & $16 \cdot 4$ & $16 \cdot 0$ & $16 \cdot 3$ & 15.5 \\
\hline Saturated fatty acids intake (\% of energy intake) & 13.5 & $13 \cdot 1$ & $12 \cdot 5$ & $12 \cdot 4$ & 11.5 \\
\hline
\end{tabular}


Table 3. Distribution of selected variables across quintiles of energy-adjusted fruit consumption in the Seguimiento Universidad de Navarra (SUN) Study

(Mean values)

\begin{tabular}{|c|c|c|c|c|c|}
\hline & Q1 (lowest) & Q2 & Q3 & Q4 & Q5 (highest) \\
\hline Participants $(n)$ & 878 & 879 & 879 & 879 & 878 \\
\hline Median fruit consumption ( $\mathrm{g} / \mathrm{d}$ ) & 114 & 174 & 263 & 406 & 726 \\
\hline Sex (\% female) & 54 & 61 & 65 & 72 & 76 \\
\hline BMI $\left(\mathrm{kg} / \mathrm{m}^{2}\right)$ & $23 \cdot 1$ & $23 \cdot 1$ & $23 \cdot 3$ & $23 \cdot 0$ & $23 \cdot 1$ \\
\hline Physical activity during leisure time in metabolic equivalents (h/week) & $19 \cdot 3$ & $19 \cdot 8$ & $19 \cdot 8$ & $20 \cdot 4$ & $20 \cdot 0$ \\
\hline Energy-adjusted alcohol consumption $(\mathrm{g} / \mathrm{d})$ & $8 \cdot 3$ & 6.5 & $6 \cdot 3$ & $5 \cdot 7$ & 4.5 \\
\hline Total fat intake ( $\%$ of energy intake) & $39 \cdot 3$ & 38.6 & $37 \cdot 7$ & $36 \cdot 2$ & 33.5 \\
\hline MUFA intake ( $\%$ of energy intake) & $16 \cdot 7$ & $16 \cdot 6$ & $16 \cdot 4$ & $15 \cdot 7$ & $14 \cdot 5$ \\
\hline Saturated fatty acids intake (\% of energy intake) & $13 \cdot 7$ & $13 \cdot 3$ & $12 \cdot 8$ & $12 \cdot 1$ & 11.0 \\
\hline
\end{tabular}

undiagnosed hypertension showed no change but the magnitude of the relationship between fruit consumption and the prevalence of undiagnosed hypertension decreased. Compared with subjects in the first quintile of vegetable consumption, the OR for those in the fifth quintile was 0.58 (95\% CI $0.36,0.91 ; P$ for trend 0.01$)$. For fruit intake, the reduction in prevalence was smaller and nonsignificant (OR 0.68; $95 \%$ CI $0.43,1.09 ; P$ for trend $0 \cdot 10)$. Fruit and vegetable consumption was correlated with other foods and nutrients. Analyses were repeated adjusting additionally for $\mathrm{K}, \mathrm{Mg}$, fibre, folic acid, total fat, or SFA intake, nuts and legumes consumption. However, estimates did not substantially change (data not shown).

When we included the sum of quintiles for fruit and vegetable consumption (range 2 to 10) in the logistic regression model as a continuous variable, the OR of undiagnosed hypertension for each additional point in this variable was 0.91 (95\% CI $0.85,0.97 ; P=0.004$ ). This was after adjusting for age, sex, BMI, physical activity, alcohol intake, Na consumption, the MUFA:SFA intake ratio, and hypercholesterolaemia. When we considered this score as a categorical variable, there was an impressive reduction of prevalence for those with the maximum score (score 10) compared with those with the minimum (score 2) (OR 0.23; $95 \%$ CI 0.10, 0.55; $P=0.001)$. But when we repeated these analyses considering as the outcome an established medical diagnosis of hypertension (i.e. those participants already labelled as 'hypertensive'), we did not observe any association with fruit and vegetable consumption.

In the analysis using the reported SBP and DBP as continuous variables, an inverse significant linear trend was evident for fruit consumption and BP. Considering SBP as the dependent variable in a multiple linear regression model, the regression coefficient for each additional $100 \mathrm{~g}$ fruit consumption was $-0.16 \mathrm{mmHg}(95 \% \mathrm{CI}-0.28$, $-0.05 ; P=0.004)$, after adjusting for age, sex and known risk factors for hypertension. For DBP, the results were similar (regression coefficient $-0.15 \mathrm{mmHg} / 100 \mathrm{~g}$ fruit; $95 \%$ CI $-0.24,-0.07 ; P<0.001)$. On the other hand, the relationship between vegetable consumption and SBP or DBP did not show a linear relationship ( $P$ values for regression coefficients 0.73 and 0.24 respectively). However, when we included a quadratic term for vegetable consumption, a significant relationship became apparent for vegetable intake both for SBP and DBP $(P<0.05$ in both cases). These coefficients were highly consistent with an inverse association for most of the range of vegetable consumption and a plateau at the highest levels of consumption. Finally, when we used the quintiles for fruit and vegetables (range 2-10) in the linear regression analysis, an inverse significant relationship with BP was observed for this variable. The regression coefficient for each additional point in the score was -0.27 (95\% CI -0.44 , $-0.10 ; P=0.002)$ for SBP and -0.27 (95\% CI -0.39 , $-0.14 ; P<0.001)$ for DBP. This implies that those with the highest fruit and vegetable consumption (score 10) had an average SBP and DBP $2.2 \mathrm{mmHg}$ lower than those with the lowest consumption (score 2 ).

The addition of an interaction term for MUFA (dichotomised at the median) and fruit-vegetable consumption (as a continuous variable) showed a marginally statistically significant improvement in the model $(P=0 \cdot 06)$. The prevalence of undiagnosed hypertension decreased with the increasing consumption of fruits and vegetables in those with a low MUFA intake, whereas the association between fruit and vegetable intake and undiagnosed hypertension was not so apparent and almost null among those with a high MUFA intake. A higher MUFA intake was also associated with a decreased prevalence of undiagnosed hypertension, especially among participants with a lower consumption of fruit and vegetables. These results were similar whether we considered absolute MUFA intake, energy-adjusted MUFA intake or percentage of MUFA from total energy intake. Given that olive oil is the main source of MUFA in Spain, we explored this same issue with energy-adjusted olive oil. The interaction term was not significant for olive oil and fruit-vegetable consumption. Finally, Table 6 reports the OR of undiagnosed hypertension according to MUFA and fruit-vegetable consumption, using individuals with the lowest intake of both fruit-vegetables and MUFA as the reference category. When we repeated these analyses with SFA or PUFA instead of MUFA intake no significant interaction was found.

\section{Discussion}

We have found an inverse relationship between fruit and vegetable consumption and the prevalence of non-previously 
Plant food consumption and blood pressure
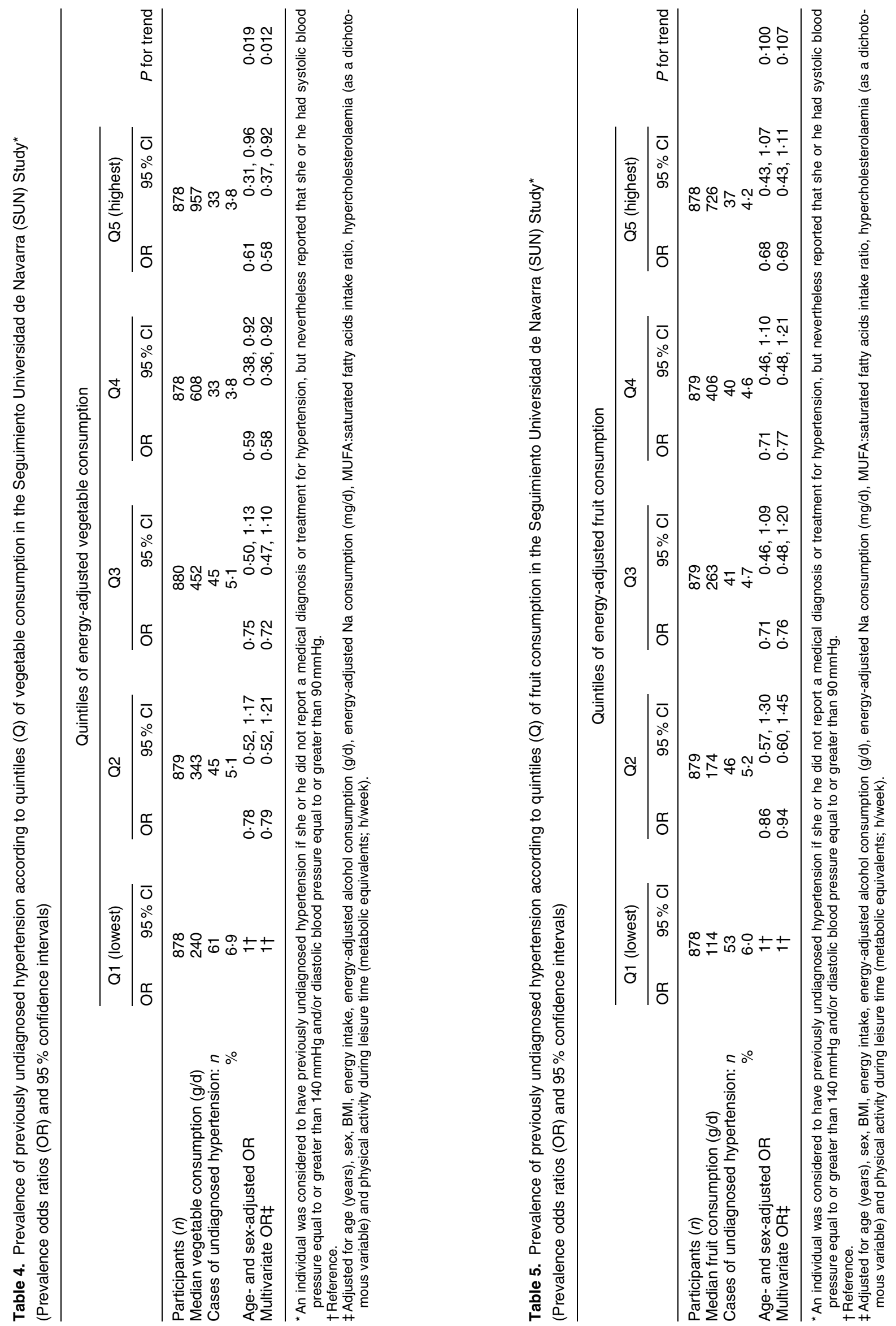
Table 6. Prevalence of previously undiagnosed hypertension according to the joint exposure to fruit and vegetable consumption (sum of quintiles of fruit and vegetable consumption) and to monounsaturated fatty acids intake in the Seguimiento Universidad de Navarra (SUN) Study*

(Prevalence odds ratios (OR) and $95 \%$ confidence intervals)

\begin{tabular}{|c|c|c|c|c|c|c|}
\hline \multirow{2}{*}{$\begin{array}{l}\text { Sum of quintiles for fruit and } \\
\text { vegetable consumption }\end{array}$} & \multicolumn{3}{|c|}{$\begin{array}{l}\text { Low MUFA intake }(<15.8 \% \text { total energy } \\
\text { intake) }(n 2198)\end{array}$} & \multicolumn{3}{|c|}{$\begin{array}{l}\text { High MUFA intake }(>15.8 \% \text { total } \\
\text { energy intake) }(n 2195)\end{array}$} \\
\hline & $n$ & OR† & $95 \% \mathrm{Cl}$ & $n$ & OR† & $95 \% \mathrm{Cl}$ \\
\hline 2 (Lowest consumption) & 178 & $1.00 \ddagger$ & & 193 & 0.35 & $0.15,0.81$ \\
\hline 3 & 177 & 0.45 & $0.21,0.99$ & 192 & 0.67 & $0.32,1.39$ \\
\hline 4 & 234 & $0 \cdot 27$ & $0.12,0.60$ & 256 & $0 \cdot 25$ & $0.11,0.60$ \\
\hline 5 & 260 & 0.46 & $0.23,0.91$ & 334 & 0.30 & $0.14,0.63$ \\
\hline 6 & 306 & 0.40 & $0.20,0.79$ & 407 & 0.33 & $0.17,0.66$ \\
\hline 7 & 306 & $0 \cdot 27$ & $0.13,0.58$ & 285 & 0.35 & $0.16,0.73$ \\
\hline 8 & 274 & 0.43 & $0.21,0.88$ & 262 & $0 \cdot 28$ & $0.12,0.65$ \\
\hline 9 & 232 & 0.23 & $0.09,0.57$ & 183 & 0.58 & $0.27,1.25$ \\
\hline 10 (Highest consumption) & 231 & 0.17 & $0.07,0.45$ & 83 & 0.08 & $0.01,0.65$ \\
\hline$P$ for trend & \multicolumn{3}{|c|}{0.001} & & \multicolumn{2}{|c|}{0.598} \\
\hline
\end{tabular}

* An individual was considered to have previously undiagnosed hypertension if she or he did not report a medical diagnosis or treatment for hypertension but nevertheless reported that she or he had systolic blood pressure equal to or greater than $140 \mathrm{mmHg}$ and/or diastolic blood pressure equal to or greater than $90 \mathrm{mmHg}$. For details of the sum of quintiles of fruit and vegetable consumption, see p. 312 .

† Adjusted for age (years), sex, BMI, energy-adjusted alcohol consumption ( $\mathrm{g} / \mathrm{d}$ ), energy-adjusted Na consumption (mg/d), hypercholesterolaemia (as a dichotomous variable) and physical activity during leisure time (metabolic equivalents; h/week). ‡Reference.

diagnosed hypertension in a Mediterranean population with a very high intake of both fat and plant-derived foods. There was a $77 \%$ reduction in the prevalence of this condition for those with the higher consumption of both fruit and vegetables compared with those situated in the lower quintiles of both food groups. This inverse relationship was also evident when considering BP as a continuous variable, with a mean SBP and DBP of $2.2 \mathrm{mmHg}$ lower for those with the highest consumption of both fruit and vegetables compared with those with the lowest intake. Interestingly, a higher MUFA intake was associated with a lower prevalence of previously undiagnosed hypertension, but only among those with a low intake of fruit and vegetables.

Results from different epidemiological studies are consistent with the present findings. Published data from the Nurses' Health Study and the Health Professionals Follow-up Study showed an inverse association between fibre and $\mathrm{Mg}$ consumption and the incidence of hypertension (Ascherio et al. 1992, 1996); both food components are very abundant in fruits and vegetables. In fact, in the Nurses' Health Study, fruit and vegetable intake, considered as a whole, was inversely related to the incidence of hypertension (Ascherio et al. 1996). In the Chicago Western Electric Study, after 8 years of follow-up, vegetable protein and an antioxidant vitamin score based on vitamin $C$ and $\beta$-carotene were inversely and significantly related to average annual change in SBP (Stamler et al. 2002).

The present results are also in accordance with those from the randomised DASH trial (Appel et al. 1997; Sacks et al. 2001). In that trial, a diet rich in fruits, vegetables, and low-fat dairy products and with reduced saturated and total fat, achieved a reduction of $5.5 \mathrm{mmHg}$ in SBP and $3.0 \mathrm{mmHg}$ in DBP, compared with a control diet. A diet rich in fruit and vegetables, but with a fat content similar to the control diet, obtained a smaller reduction in BP levels. These results were obtained in a controlled environment, with specifically designed diets.
Interestingly, the present results are derived from a freeliving population, evidencing a similar effect of a high consumption of fruits and vegetables on BP in the general public. Moreover, a high fat intake was present in our population. The adoption of a diet rich in fruits and vegetables, even in the context of a high fat intake, could potentially shift the population distribution of BP downward, reducing the occurrence of BP-related cardiovascular disease.

The recent PREMIER Clinical Trial (Writing Group of the PREMIER Collaborative Research Group, 2003) documented that individuals with above-optimal BP can benefit from recommendations aimed to attain a similar diet to that used in the DASH trial. The individuals obtained BP levels lower than those derived from more limited lifestyle modifications (weight reduction, Na restriction, increased physical activity), although the decrease was not significant. In the present study, we found that in a different and non-controlled setting some elements of the DASH diet had a similar effect on BP. Moreover, we assessed individuals whose usual diets contain a very high intake of both fat and plantderived nutrients, supporting the view that it is possible and realistic to follow a diet with these characteristics.

Although the SUN Study is a prospective cohort study, the reported results are derived from a cross-sectional analysis. This kind of analysis has potential drawbacks, including the difficulty to distinguish cause and effect. However, we have excluded from our analysis individuals with medically diagnosed hypertension (labelled 'hypertensives'). Diets in these individuals are probably different due to their lifestyle modifications derived from diagnosis and clinical advice from their physicians. In a study conducted in a Spanish population, awareness of hypertension was associated with an increase in the consumption of plant foods (Tormo et al. 2000). Including only individuals not previously labelled as diseased makes less likely a disease-driven change in diet and the reverse causation bias. In this way, it is possible to assume that the reported diet 
is a good approximation to past and present diet. Anyway, diet changes derived from the awareness of hypertension would include an increase in fruit and vegetable consumption, thus obscuring the association between the intake of these foods and BP. We have observed an inverse relationship despite this possible bias.

There could be other factors associated both with BP and the intake of fruit and vegetables that might confound the present results. In our analysis we have adjusted for the main known risk factors for hypertension (alcohol and $\mathrm{Na}$ intake, level of physical activity, and overweight). Besides, our population is relatively homogeneous, all being university graduates, allowing a better comparability of subjects (Rothman \& Greenland, 1998).

Other nutrients and foods, such as nuts, legumes, whole grain, fibre, and $\mathrm{Mg}$, are highly correlated with fruit and vegetable intake. Analysis adjusted for these variables did not change our estimations. In fact, these foods were not independently associated with the prevalence of undiagnosed hypertension.

An obvious limitation of the present study is the high percentage of non-response. This could be seen as a selection bias. Actually, non-respondents were younger and with a different dietary profile compared with respondents. However, it is conceivable that these discrepancies did not account for a diverse relationship between plant-food consumption and undiagnosed hypertension in both groups of subjects.

Prevalence of hypertension in the Spanish adult general population is about $45 \%$ (Banegas et al. 1998), although less than one half of the affected is aware of his or her condition. The awareness of hypertension has increased in Spain from 67\%, in 1988, to 76\%, in 1996 (Sans et al. 2001). Furthermore, hypertension awareness changes across different groups. For example, women, older individuals and those with a higher educational or socio-economic level report more accurate $\mathrm{BP}$ values (Colditz et al. 1986; Goldman et al. 2003). In the SUN Study, the selfreported medical diagnosis of hypertension at baseline was $14.9 \%$. SUN participants are relatively young and well educated, which may account for a lower prevalence. However, this does not affect the reported results, given that the objective of the present study was not to estimate the prevalence of hypertension in the general population, but to assess the relationship between diet and BP. Using a highly selected population increases the internal validity of the study, because of a higher awareness and a higher reliability of self-reported data.

Information from the dietary intake obtained through a food-frequency questionnaire is usually a good correlate of usual diet. Nonetheless, a certain degree of misclassification always occurs. This problem would shift estimations towards the null value. So, the real association between BP and fruit and vegetable intake is probably greater than that reported in the present study. In this case, the validation study for the questionnaire used in the present study showed a fair reliability (Martín-Moreno et al. 1993).

It is possible that the overall plant-food-derived components of diet (including vegetable oils) influence the prevalence of hypertension. The biological pathways for the effect of plant-derived foods on BP levels are diverse.
$\mathrm{K}$ is one of the most important nutrients in this relationship (He \& MacGregor, 2001). In contrast, we were not able to show a significant association between $\mathrm{K}$ and $\mathrm{BP}$; when we adjusted for $\mathrm{K}$ intake in the analysis, there were no substantial changes in the estimates for the association of BP with fruit and vegetable consumption. We acknowledge that this can also be due to inaccurate information about the specific $\mathrm{K}$ content of some foods leading to some degree of measurement error in $\mathrm{K}$ intake, which may conceal the actual relationship between $\mathrm{K}$ and lower BP. However, an alternative explanation is that other plant-food components are also implicated in the protection against hypertension such as antioxidants, present in high concentrations in fruits and vegetables. Indeed, it has been shown that $100 \mathrm{~g}$ fresh apples may have an antioxidant activity as high as $1500 \mathrm{mg}$ ascorbic acid (Eberhardt et al. 2000). Vascular oxidative stress may also be implicated in the pathophysiology of hypertension, resulting in impaired endothelium-dependent vasodilatation (McIntyre et al. 1999). In patients with essential hypertension, the infusion of an antioxidant such as ascorbic acid normalises endothelial function by restoring the NO-mediated vasodilatation of the endothelium (Sowers, 2002). In a controlled feeding trial, the consumption of antioxidants from vegetable sources has produced a reduction in the endothelial impairment due to a high-fat meal (Esposito et al. 2003). However, the overall effect of ascorbic acid and other antioxidants on BP is far from being resolved (Kim et al. 2002; Mullan et al. 2002; Svetkey \& Loria, 2002). All these pathways represent complementary or alternative mechanisms to $\mathrm{K}$ which might explain the protection of fruit and vegetable intake against hypertension.

There is also biological plausibility for the reported effect of MUFA intake on BP. In some studies, it has been observed that MUFA intake decreases BP levels (Ruiz-Gutiérrez et al. 1996) and reduces the need for anti-hypertensive medication in hypertensives (Ferrara et al. 2000). The effect of MUFA on BP could be mediated through a decrease in insulin resistance (Salas et al. 1999).

Interestingly, we have observed that the association of fruit and vegetable consumption with undiagnosed hypertension was different according to the level of MUFA consumption (effect modification). The effect of each dietary component was greater when there was a low consumption of the other one. This is consistent with a causal effect of these foods and nutrients on BP; individuals at higher risk receive greater benefit from exposure to a preventive factor, whereas those with lower risk due to other healthy lifestyles do not exhibit additional benefit from protective exposures. However, these results must be considered cautiously given that the interaction term was marginally statistically significant and could be a false positive result.

In summary, we have found that a diet rich in fruits and vegetables is associated with a lower BP level in a Mediterranean free-living population with an abundant intake of both fat and plant-derived foods. However, it remains unexplored the potential subgroups which especially benefited from these food groups. The consideration of realistic dietary patterns that the population usually and easily may adopt will bring a more practical approach to the prevention of hypertension through diet, since nutrients and 
foods are consumed in association. The follow-up of a cohort with a high consumption of foods supposed to be protective will give rise to sound scientific evidence.

\section{Acknowledgements}

We are indebted to the participants of the SUN Study for their continued cooperation and participation. The Department of Health of the Navarre Regional Government and Spanish Ministry of Health (Fondo de Investigaciones Sanitarias, projects 01/0619 and G03/140, Red Temática de Dieta y Enfermedad Cardiovascular) are gratefully acknowledged for supporting the present study. We would like to thank the other members of the SUN Study Group: M. Seguí-Gómez, M. Delgado-Rodríguez, F. Guillén-Grima, I. Aguinaga, C. Rubio, A. Sánchez-Villegas, A. Martí, M. Hernández, M. Perepérez, N. Egüés, M. Bes, R. Pajares, J. Gómez-Aracena and M. Muñoz.

\section{References}

Ainsworth BE, Haskell WL, Whitt MC, et al. (2000) Compendium of physical activities: an update of activity codes and MET intensities. Med Sci Sports Exerc 32, S498-S504.

Appel LJ, Moore TJ, Obarzanek E, et al. (1997) A clinical trial of the effects of dietary patterns on blood pressure. $N$ Engl J Med 336, 1117-1124.

Ascherio A, Hennekens CH, Willett WC, Sacks FM, Rosner B, Manson JE, Witteman JCM \& Stampfer MJ (1996) Prospective study of nutritional factors, blood pressure, and hypertension among US women. Hypertension 27, 1065-1072.

Ascherio A, Rimm EB, Giovanucci EL, Colditz GA, Rosner B, Willett WC, Sacks FM \& Stampfer MJ (1992) A prospective study of nutritional factors and hypertension among US men. Circulation 86, 1475-1484.

Banegas JR, Rodríguez-Artalejo F, de la Cruz Troca JJ, GuallarCastillón P \& del Rey Calero J (1998) Blood pressure in Spain. Distribution, awareness, control, and benefits of a reduction in average pressure. Hypertension 32, 998-1002.

Chobanian AV, Bakris GL, Black HR, et al. (2003) The seventh report of the Joint National Committee on Prevention, Detection, Evaluation, and Treatment of High Blood Pressure: the JNC 7 report. JAMA 289, 2560-2571.

Colditz GA, Martin P, Stampfer MJ, Willett WC, Sampson L, Rosner B, Hennekens CH \& Speizer FE (1986) Validation of questionnaire information on risk factors and disease outcomes in a prospective cohort study of women. Am J Epidemiol 123, 894-900.

De Lorgeril M, Salen P, Martin JL, Monjaud I, Delaye J \& Mamelle N (1999) Mediterranean diet, traditional risk factors, and the rate of cardiovascular complications after myocardial infarction: final report of the Lyon Diet Heart study. Circulation 99, 779-785.

Eberhardt MV, Lee CY \& Liu RH (2000) Antioxidant activity of fresh apples. Nature 405, 903-904.

Esposito K, Nappo F, Giugliano F, Giugliano G, Marfella R \& Giugliano D (2003) Effect of dietary antioxidants on postprandial endothelial dysfunction induced by a high-fat meal in healthy subjects. Am J Clin Nutr 77, 139-143.

Ferrara LA, Raimondi AS, d'Episcopo L, Guida L, Dello Russo A \& Marotta T (2000) Olive oil and reduced need for antihypertensive medications. Arch Intern Med 160, 837-842.

Goldman N, Lin I-F, Weinstein M \& Lin Y-H (2003) Evaluating the quality of self-reports of hypertension and diabetes. J Clin Epidemiol 56, 148-154.
He FJ \& MacGregor GA (2001) Beneficial effects of potassium. BMJ 323, 497-501.

Hermansen K (2000) Diet, blood pressure and hypertension. Br J Nutr 83, S113-S119.

Hosmer DW \& Lemeshow S (2000) Applied Logistic Regression, 2nd ed. New York: Wiley.

Kim MK, Sasaki S, Sasazuki S, Okubo S, Hayashi M \& Tsugane S (2002) Lack of long-term effect of vitamin C supplementation on blood pressure. Hypertension 40, 797-803.

McIntyre M, Bohr DF \& Dominiczak AF (1999) Endothelial function in hypertension: the role of superoxide anion. Hypertension 34, 539-545.

Martínez-González MA, Alonso A, de Irala-Estévez J \& Fernández-Jarne E (2003) What is protective in the Mediterranean diet? Atherosclerosis 166, 405-407.

Martínez-González MA, Martínez JA, Hu FB, Gibney MJ \& Kearney J (1999) Physical inactivity, sedentary lifestyles, and obesity in the European Union. Int $J$ Obes Relat Metab Disord 23, 1192-1201.

Martínez-González MA \& Sánchez-Villegas A (2004) The emerging role of Mediterranean diets in cardiovascular epidemiology: monounsaturated fats, olive oil, red wine or the whole pattern? Eur J Epidemiol 19, 9-13.

Martínez-González MA, Sánchez-Villegas A, de Irala-Estévez J, Martí A \& Martínez JA (2002) Mediterranean diet and stroke: objectives and design of the SUN project. Nutr Neurosci 5, 65-73.

Martín-Moreno JM, Boyle P, Gorgojo L, Maisonneuve P, Fernández-Rodríguez JC, Salvini S \& Willett WC (1993) Development and validation of a food frequency questionnaire in Spain. Int J Epidemiol 22, 512-519.

Mataix Verdú J (2003) Tabla de Composición de Alimentos Espanoles, 4th ed. Granada, Spain: Universidad de Granada.

Mullan BA, Young IS, Fee H \& McCance DR (2002) Ascorbic acid reduces blood pressure and arterial stiffness in type 2 diabetes. Hypertension 40, 804-809.

Robertson RM \& Smaha L (2001) Can a Mediterranean-style diet reduce heart disease? Circulation 103, 1821-1822.

Rothman KJ (2002) Types of epidemiologic study. In Epidemiology: An Introduction, pp. 57-93 [KJ Rothman, editor]. New York: Oxford University Press.

Rothman KJ \& Greenland S (1998) Modern Epidemiology, 2nd ed. Philadelphia, PA: Lippincott-Raven.

Ruiz-Gutiérrez V, Muriana FJ, Guerrero A, Cert AM \& Villar J (1996) Plasma lipids, erythrocyte membrane lipids and blood pressure of hypertensive women after ingestion of dietary oleic acid from two different sources. $J$ Hypertens 14, $1483-1490$.

Sacks FM, Svetkey LP, Vollmer WM, et al. (2001) Effects on blood pressure of reduced dietary sodium and the Dietary Approaches to Stop Hypertension (DASH) diet. $N$ Engl $J$ Med 344, 3-10.

Salas J, López Miranda J, Jansen S, et al. (1999) The diet rich in monounsaturated fat modifies in a beneficial way carbohydrate metabolism and arterial pressure (in Spanish). Med Clin (Barc) 113, 765-769.

Sánchez-Villegas A, Delgado-Rodríguez M, Martínez-González MA \& de Irala-Estévez J (2003) Gender, age, socio-demographic and lifestyle factors associated with major dietary patterns in the Spanish project SUN (Seguimiento Universidad de Navarra). Eur J Clin Nutr 57, 285-292.

Sans S, Paluzie G, Balana L, Puig T \& Balaguer-Vintró I (2001) Trends in prevalence, awareness, treatment and control of arterial hypertension between 1986 and 1996: the MONICA-Catalonia study (in Spanish). Med Clin (Barc) 117, 246-253.

Singh RB, Dubnov G, Niaz MA, Ghosh S, Singh R, Rastogi SS, 
Manor O, Pella D \& Berry EM (2002) Effect of an Indo-Mediterranean diet on progression of coronary artery disease in high risk patients (Indo-Mediterranean Diet Heart study): a randomised single-blind trial. Lancet 360, 1455-1461.

Sowers JR (2002) Hypertension, angiotensin II, and oxidative stress. N Engl J Med 346, 1999-2001.

Stamler J, Liu K, Ruth KJ, Pryer J \& Greenland P (2002) Eightyear blood pressure change in middle-aged men: relationship to multiple nutrients. Hypertension 39, 1000-1006.

Svetkey LP \& Loria CM (2002) Blood pressure effects of vitamin C: what's the key question? Hypertension 40, 789-791.

Tormo MJ, Navarro C, Chirlaque MD \& Barber X (2000) Is there a different dietetic pattern depending on self-knowledge of high blood pressure? Eur J Epidemiol 16, 963-971.

Trichopoulou A, Costacou T, Barnia C \& Trichopoulos D (2003)
Adherence to a Mediterranean diet and survival in a Greek population. N Engl J Med 348, 2599-2608.

Whelton PK, He J, Appel LJ, et al. (2002) Primary prevention of hypertension: clinical and public health advisory from the National High Blood Pressure Education Program. JAMA 288, 1882-1888.

Willett WC (1998) Nutritional Epidemiology, 2nd ed. New York: Oxford University Press.

Willett WC, Sacks FM, Trichopoulou A, Drescher G, Ferro-Luzzi A, Helsing E \& Trichopoulos D (1995) Mediterranean diet pyramid: a cultural model for healthy eating. Am J Clin Nutr $\mathbf{6 1}$, Suppl., 1402S-1406S.

Writing Group of the PREMIER Collaborative Research Group (2003) Effects of comprehensive lifestyle modification on blood pressure control: main results of the PREMIER Clinical Trial. JAMA 289, 2083-2093. 